\title{
Study on Protection Effectiveness of Early Streamer Emission Lightning Protection Systems
}

\author{
Yen-Hong A. Chen ${ }^{a}$, KaiJan Lin ${ }^{b^{*}}$ and Yu-Chu M. Lic \\ Department of Mechanical Engineering, Southern Taiwan University of Science and Technology, \\ Taiwan, ROC \\ ${ }^{b}$ kjlin@stust.edu.tw
}

Keywords: Early Streamer Emission, ESE, Lightning Arrester.

\begin{abstract}
A new early streamer emission (ESE) lightning air terminal system is designed, fabricated. By comparing the arrested artificial lightning numbers of ESE and traditional Franklin terminals in laboratory, the effectiveness of ESE arrester is validated. A Tesla Coil with controlled triggering function generator is used to produce simulated lightning strikes. Both terminals are placed with same distance to the TC during the testing processes and exchanging their positions does not influence the observed results. The tested data validate the effectiveness of the ESE terminal under the laboratory environment.
\end{abstract}

\section{Introduction}

Lightning strike appears suddenly and is unpredictable in advance. It is a kind of physical phenomenon classified to electrostatic discharging caused by storm. Lightning strikes to the earth ground could cause damage for their high temperature, high electric current all in a short time. Humans or animals could be killed and properties like buildings, equipment, buildings systems, electronics and vehicles will be serious damage extensively as lightning striking happened.

Summer seasons in Taiwan, a number of afternoon thunderstorms and typhoons emerge every year; people get killed and properties get damaged by lightning strike [1] are not unfamiliar. The unpredictable and fatal lightning strikes are more regular as the trend of global warming and lightning strike events will increase due to climate change. According to the official statistics from Taiwan Power Company[2], during the second and third quarter in 2010, the lightning strikes in Taiwan were more than 20,000 times. The impressive striking number indicates the need of devices with effective protection under lightning striking in Taiwan, not only for lives of people but also the properties of natives.

In order to effectively prevent possible injury by lightning [3], this study has designed and builds an ESE terminal and makes tests for comparing effectiveness with Franklin terminal.

\section{Overview of The Experiments}

An ESE air terminal is designed and carried out. The conducting parts of the terminal are made of stainless steels 304 . The height of the assembled terminal is $170 \mathrm{~cm}$ and the overall weight is less than 25 kilogram. The configuration of our testing system is shown in Fig. 1. A homemade Tesla coil (TC) discharging by powering ac voltage up to $650 \mathrm{kV}$ is used to simulate lightning strikes. The TC is put on a metallic grounded plane. The tested ESE and Franklin terminals are fixed on the same plane and keep good contact with the plane. A pointed aluminum rod is placed on the top of TC in order to discharge strikes from the same position during all the tests. The relative positions of ESE terminal, brass-made Franklin terminal (denoted by conventional lightning rod CLR) and TC are shown in Fig. 2. The tip shape of the pointed aluminum rod is kept conformity with [4]. The spark length made by TC is no more than $40 \mathrm{~cm}$. The two terminals are kept with the same height and are $0.5 \mathrm{~m}$ apart horizontally. The central lightning source above each terminal keeps the same distance of $0.3 \mathrm{~m}$ to each terminal. Such arrangement of relative position is to make the terminals within the striking distance of the lightning and prevents the influence from static electric field of the ground plane. Two 
counters for counting arrested strikes of the terminals are connected to the separated ground lines of the terminals. The controlled discharging of TC simulated lightning strikes is triggered by a function generator (FG) with periodic pulses of voltage serial signals.

In each test configuration, a total of 50 strikes from TC triggered by the function generator are recorded. The period between continuous triggering signals is assigned from one second to five seconds. The arrested impulse strike on the terminal is recorded by a homemade counting system includes an AC current sensor, an amplifier and an electromagnetic counter.

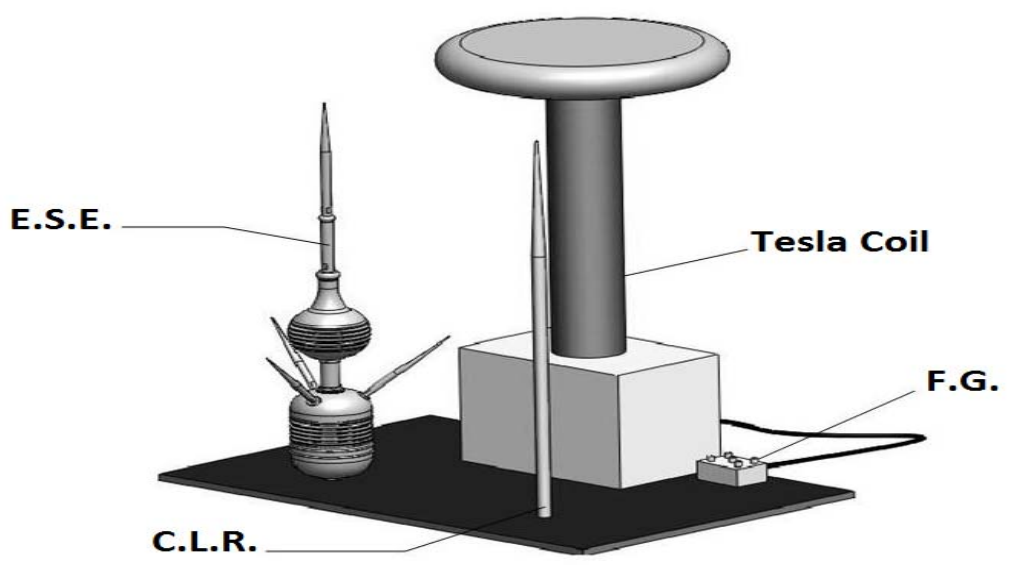

Figure 1. The configuration of the testing system for comparing protection effectiveness.

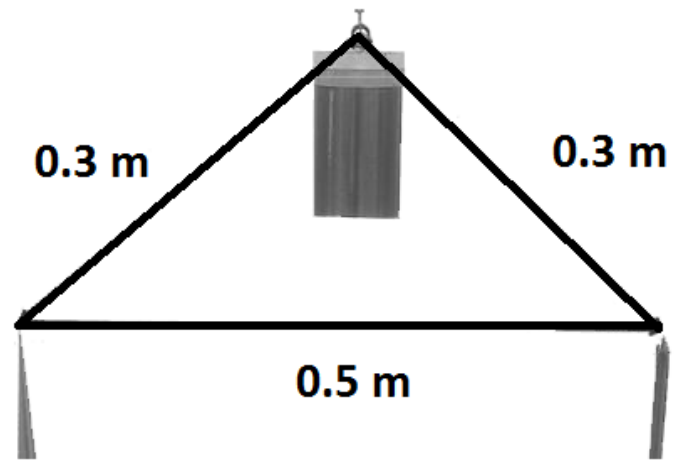

E.S.E

C.L.R.

Figure 2. The relative positions of ESE terminal, CLR and TC.

\section{Results and Discussion}

Fig. 3 shows the result of 15 tests comparing the arrested lightning strikes by each terminal. Each test contains 50 strikes in total. Some strikes reach none of the terminals is recorded also. Based on the result, the ratio of the number caught by the ESE terminal to the number caught by CLR is $28: 22$ in average for all 15 runs. The ESE terminal shows better probability to catch the strikes on all tests and the triggering time period from one to five seconds makes no apparent difference. Change the places of terminals with each other, result the same test result. 


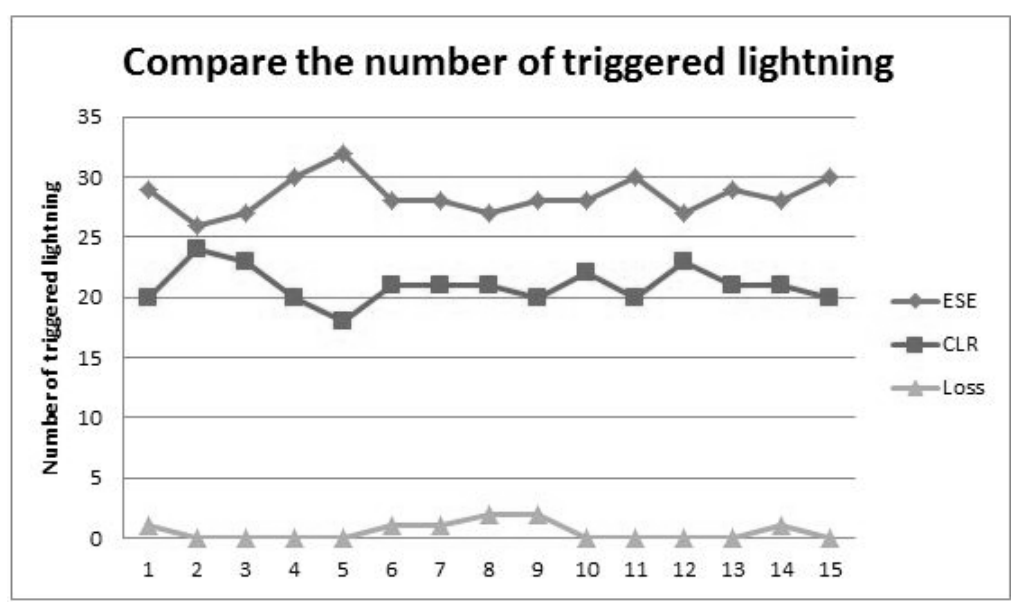

Figure 3. The result of arrested lightning strikes of ESE terminal and CLR. There are 50 strikes delivered from TC for each run in total.

\section{Conclusions}

It is claimed that the protection radius of the ESE terminal is superior to CLR. An effectiveness test of comparing the ESE terminal and CLR is presented in this work. The ESE lightning terminal is designed and fabricated in out laboratory to test the effectiveness with conventional lightning rod. The simulated lightning strikes on the terminals are produced from a home-made Tesla coil with electrical resonant transformer circuit designed by inventor Nikola Tesla around 1891. The result of counting arresting strikes number on both terminals supports the ESE terminal working better with much opportunity on arresting lightning strikes.

\section{References}

[1] http://www.libertytimes.com.tw/2010/new/sep/14/today-center1.htm, The Liberty Times e-news.

[2] http://www.taipower.com.tw/, Taiwan Power Company.

[3] Karthick Srinivasan, Jason GU, "Lightning as Atmospheric Electricity", IEEE CCECE/CCGEI, Ottawa, pp. 2258-2261, May. 2006.

[4] F. A. M. Rizk, "Modeling of Lightning Exposure of Sharp and Blunt Rods", IEEE Transactions on Power Delivery, Vol. 25, No. 4, Oct. 2010.

[5] http://www.scribd.com/doc/20738563/NFC-17-102. 